\title{
Isomerization of $\alpha$-pinene oxide over cerium and tin catalysts: Selective synthesis of trans-carveol and trans-sobrerol
}

\author{
Vinícius V. Costa ${ }^{a}$, Kelly A. da Silva Rocha ${ }^{b}$, Líniker F. de Sousa ${ }^{b}$, Patricia A. Robles-Dutenhefner ${ }^{\mathrm{b}}$, \\ Elena V. Gusevskaya ${ }^{a, *}$ \\ a Departamento de Química, Universidade Federal de Minas Gerais, 31270-901, Belo Horizonte, MG, Brazil \\ b Departamento de Química, Universidade Federal de Ouro Preto, 35400-000, Ouro Preto, MG, Brazil
}

\section{A R T I C L E I N F O}

\section{Article history:}

Received 3 March 2011

Received in revised form 9 May 2011

Accepted 22 May 2011

Available online 27 May 2011

\section{Keywords:}

Isomerization

$\alpha$-Pinene oxide

Solvent effect

Tin catalysts

Cerium catalysts

\begin{abstract}
A B S T R A C T
A remarkable effect of the solvent nature on the acid catalyzed transformation of $\alpha$-pinene oxide allowed direction of the reaction to either trans-carveol or trans-sobrerol. Each of these highly valuable compounds was obtained in nearly $70 \%$ yield using an appropriate polar solvent, whose basicity affected strongly the product distribution. In acetone, a weakly basic solvent, the reaction over heterogeneous sol-gel $\mathrm{Sn} / \mathrm{SiO}_{2}$ or $\mathrm{Ce} / \mathrm{SiO}_{2}$ catalysts gave mainly trans-sobrerol. No leaching of active components occurs under the reaction conditions and the catalysts can be recovered and reused. On the other hand, in more basic solvent, i.e., dimethylacetamide, the reaction was essentially directed to trans-carveol. Due to the leaching problems with $\mathrm{Sn} / \mathrm{SiO}_{2}$ and $\mathrm{Ce} / \mathrm{SiO}_{2}$ materials, the synthesis of trans-carveol was performed under homogeneous conditions using $\mathrm{CeCl}_{3}$ or $\mathrm{SnCl}_{2}$ as catalysts with a catalyst turnover number up to ca. 1200. The method represents one of the few examples of the synthesis of isomers from $\alpha$-pinene oxide, other than campholenic aldehyde, with a sufficient for practical usage selectivity.
\end{abstract}

(C) 2011 Elsevier B.V. All rights reserved.

\section{Introduction}

Terpenic compounds, in general, are an important renewable feedstock for the flavor and fragrance industry. Their oxygenated derivatives usually show interesting organoleptic properties and form one of the most important groups of fragrance ingredients $[1,2]$. $\alpha$-Pinene, a major constituent of turpentine oils obtained from coniferous trees, is a particularly valuable raw material in the production of synthetic substitutes for natural aromas. The epoxidation of $\alpha$-pinene provides $\alpha$-pinene oxide, which can be converted by acid-catalyzed transformations in various expensive ingredients for the flavor industry, such as campholenic aldehyde, trans-carveol, trans-sobrerol and trans-pinocarveol.

$\alpha$-Pinene oxide is highly reactive in the presence of acid and can result in a great variety of products. In one of the previous works, over 200 compounds formed from $\alpha$-pinene oxide at temperatures above $100^{\circ} \mathrm{C}$ have been found [3]. Therefore, the development of the reaction which is selective for one particular product is a very challenging task. Most of the studies have been focused on the synthesis of campholenic aldehyde, which is used for the manufacture

\footnotetext{
* Corresponding author. Tel.: +55 31 34095741; fax: +55 3134095700 .

E-mail address: elena@ufmg.br (E.V. Gusevskaya).
}

of sandalwood-like fragrances [4]. Campholenic aldehyde can be obtained from $\alpha$-pinene oxide with a sufficient for practical applications selectivity [5-7], whereas all other compounds are usually produced as minor products with less than $25 \%$ selectivities.

We have recently studied the application of heteropoly acid $\mathrm{H}_{3} \mathrm{PW}_{12} \mathrm{O}_{40}$ (PW) for the liquid-phase isomerization of $\alpha$-pinene oxide in various solvents [8,9]. A remarkable effect of solvent polarity and basicity on the chemoselectivity of this reaction was found, which allowed a selective synthesis of various valuable fragrance compounds with reasonable selectivities. In cyclohexane, a non-polar solvent, 70\% selectivity to campholenic aldehyde was obtained on a heterogeneous silica-supported PW catalyst, with a combined yield for campholenic aldehyde and trans-carveol being nearly quantitative. On the other hand, the use of polar solvents allowed switching the reaction pathway to the formation of compounds with a para-menthenic skeleton, such as trans-carveol and trans-sobrerol. Polar basic solvents, such as dimethylacetamide, favored the formation of trans-carveol; whereas polar weakly basic solvents, such as acetone, promoted the formation of trans-sobrerol [9].

The highest previously reported yield of trans-carveol (45\%), an expensive constituent of the Valencia orange essence oil used in perfume bases and food flavor compositions, was obtained using acidic molecularly imprinted polymers as protic catalysts in equimolar to $\alpha$-pinene oxide amounts [10]. trans-Sobrerol, a wellknown mucolytic drug, presently attracts increasing attention for 


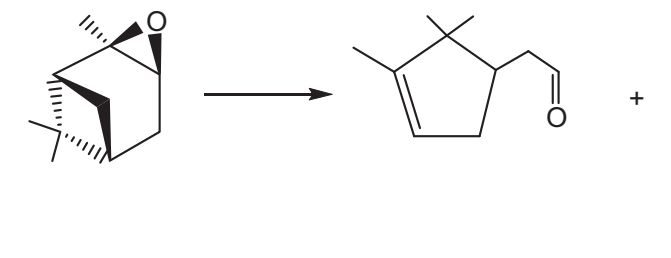

1

$\alpha$ - pinene oxide
2

campholenic aldehyde

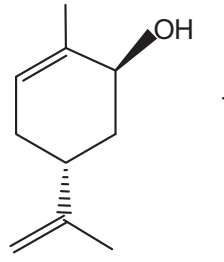

3

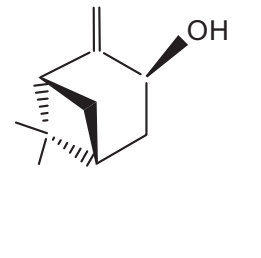

4

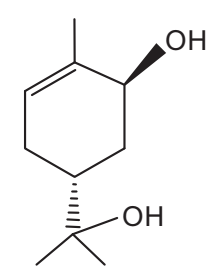

5 trans-carveol trans-pinocarveol

Scheme 1. Some products of the acid-catalyzed transformations of $\alpha$-pinene-oxide.

its other important biological activities [11]. The highest previously reported yield of trans-sobrerol (30\%) was obtained in the reflux dichloroethane solutions of $\alpha$-pinene oxide and catalytic amounts of $\mathrm{TiCl}_{4}[12]$. Although we found that the PW catalyst could promote the formation of trans-carveol and trans-sobrerol in higher than 70\% yield each using an appropriate polar solvent [9], the reactions were performed under homogeneous conditions due to high solubility of PW in polar solvents. Nevertheless, these results encouraged us to work further with the isomerization of $\alpha$-pinene oxide in order to develop heterogeneous catalytic processes for the selective synthesis of the products other than campholenic aldehyde, in particular, trans-carveol and trans-sobrerol.

Within our program aimed at adding value to natural ingredients of renewable essential oils [13-16] we decided to prepare cerium and tin containing molecular sieves and used them as acid catalysts for the isomerization of $\alpha$-pinene oxide in polar solvents. The catalysts have been synthesized through a direct hydrothermal sol-gel technique which usually affords more stable to leaching materials than conventional impregnation methods due to higher levels of metal incorporation in a silica framework.

In the present work, we report a simple and efficient synthesis of several highly valuable fragrance and/or pharmaceutical compounds through the liquid-phase isomerization of $\alpha$-pinene oxide in the presence of cerium and tin catalysts. A remarkable effect of solvent basicity on chemoselectivity allowed direction of the reaction to either trans-carveol or trans-sobrerol, which were obtained in nearly $70 \%$ yields.

\section{Experimental}

\subsection{Catalyst preparation and characterization}

The $\mathrm{Sn} / \mathrm{SiO}_{2}$ and $\mathrm{Ce} / \mathrm{SiO}_{2}$ catalysts were prepared by a sol-gel method using tetraethoxysilane $(14.90 \mathrm{~g}, \mathrm{TEOS}, 99 \%$, Sigma-Aldrich) and $\mathrm{SnCl}_{2} \times 2 \mathrm{H}_{2} \mathrm{O}(0.90 \mathrm{~g}$, Sigma-Aldrich $)$ or $\mathrm{CeCl}_{3} \times 7 \mathrm{H}_{2} \mathrm{O}(1.44 \mathrm{~g}$, Sigma-Aldrich), respectively, as precursors. The sol was obtained from a TEOS/ethanol/water mixture in a $1 / 3 / 10$ molar ratio with the addition of $\mathrm{HCl}$ and $\mathrm{HF}$ (up to $\mathrm{pH} 2.0$ ) as catalysts. The samples were dried at $110^{\circ} \mathrm{C}$ for $24 \mathrm{~h}$ and thermally treated for $2 \mathrm{~h}$ at $500{ }^{\circ} \mathrm{C}$ in air. A pure $\mathrm{SiO}_{2}$ to be tested in blank reactions was prepared by the same procedure without the addition of cerium or tin chlorides. The determination of total tin and cerium contents was performed by inductively coupled plasma atomic emission spectrometry (ICP-AES) on a Spectro Ciros CCD instrument.

The textural characteristics of the catalysts were determined from nitrogen adsorption isotherms (Autosorb-Quantachrome NOVA-1200 instrument, nitrogen, $\left.-196^{\circ} \mathrm{C}\right)$. Samples were outgassed for $2 \mathrm{~h}$ at $300^{\circ} \mathrm{C}$ before analysis. Specific surface areas were determined by the Brunauer-Emmett-Teller (BET) equation. Average pore diameters were determined by the Barrett-Joyner-Halenda (BJH) method.

\subsection{Catalytic experiments}

The reactions were carried out in a glass reactor equipped with a magnetic stirrer at $5-140^{\circ} \mathrm{C}$. In a typical run, a mixture of $\alpha$-pinene oxide $(0.122-0.365 \mathrm{~g}, 0.8-2.4 \mathrm{mmol})$, dodecane $(110 \mu \mathrm{L}$, $0.5 \mathrm{mmol}$, internal standard) and the solid catalyst (0.03-0.12 g of $\mathrm{Ce} / \mathrm{SiO}_{2}, \mathrm{Sn} / \mathrm{SiO}_{2}$ or $\mathrm{SiO}_{2}$ ) or soluble metal chloride $(5-20 \mu \mathrm{mol}$ of $\mathrm{CeCl}_{3} \times 7 \mathrm{H}_{2} \mathrm{O}$ or $\left.\mathrm{SnCl}_{2} \times 2 \mathrm{H}_{2} \mathrm{O}\right)$ in a specified solvent $(5 \mathrm{~mL})$ was intensively stirred under air at a specified temperature. The reaction progress was followed by gas chromatography (GC) using a Shimadzu 17 instrument fitted with a Carbowax $20 \mathrm{M}$ capillary column and a flame ionization detector. At appropriate time intervals, aliquots were taken and analyzed by GC. The GC mass balance, product selectivities and yields were calculated based on the substrate charged using dodecane as internal standard. The difference was attributed to the formation of oligomers, which were not GC determinable.

Catalyst recycling experiments were performed as follows: after the reaction, the catalyst was centrifuged, washed with acetonitrile and then with cyclohexane and reused. To control metal leaching, the catalyst was removed at the reaction temperature and the solution was allowed to react further.

The structures of products 2-5 were confirmed by GC/MS (Shimadzu QP2010-PLUS instrument, $70 \mathrm{eV}$ ) by comparison with authentic samples. Major products were isolated by column chromatography (silica) and identified by ${ }^{1} \mathrm{H}$ and ${ }^{13} \mathrm{C}$ NMR spectroscopy (Bruker DRX-400, tetramethylsilane, $\mathrm{CDCl}_{3}, \mathrm{COSY}, \mathrm{HMQC}, \mathrm{DEPT}$ and NOESY experiments).

Spectroscopic data for campholenic aldehyde (2) and trans-carveol (3) have been reported in our previous work [8].

Spectroscopic data for trans-pinocarveol (4): MS ( $m / z /$ rel.int.): $134 / 27\left[\mathrm{M}^{+}-\mathrm{H}_{2} \mathrm{O}\right], 119 / 53,109 / 33,105 / 20,95 / 20,93 / 32,92 / 100$, $91 / 80,83 / 78,81 / 38,79 / 32,77 / 20,70 / 92,69 / 50,67 / 22,55 / 90,53 / 20$.

Spectroscopic data for trans-sobrerol (5) have been reported previously [9].

\section{Results and discussion}

The results of the elemental analysis of the prepared solid materials, their BET surface areas, average pore sizes, and pore volumes are presented in Table 1 . Both $\mathrm{Ce} / \mathrm{SiO}_{2}$ and $\mathrm{Sn} / \mathrm{SiO}_{2}$ samples exhibited the isotherms characteristic of mesoporous materials and relatively higher specific surface areas (297 and $366 \mathrm{~m}^{2} \mathrm{~g}^{-1}$, respectively). The materials were tested as heterogeneous acid catalysts in the liquid-phase isomerization of $\alpha$-pinene oxide in various solvents. Transformations of $\alpha$-pinene oxide (1) under acidic conditions can result in campholenic aldehyde (2), transcarveol (3), trans-pinocarveol (4) and trans-sobrerol (5) shown in Scheme 1 , as well as in numerous other products. In the present work, our efforts were directed to achieve high selectivity to any other than campholenic aldehyde product and to perform the process under heterogeneous conditions, i.e., in the absence of leaching 
Table 1

Elemental analysis data and textural properties of the $\mathrm{Ce} / \mathrm{SiO}{ }_{2}$ and $\mathrm{Sn} / \mathrm{SiO}{ }_{2}$ catalysts.

\begin{tabular}{|c|c|c|c|c|}
\hline Sample & $\begin{array}{l}\text { Ce or Sn } \\
\text { content (wt\%) }\end{array}$ & $\begin{array}{l}\text { BET surface } \\
\text { area }\left(\mathrm{m}^{2} \mathrm{~g}^{-1}\right)\end{array}$ & $\begin{array}{l}\text { Total pore } \\
\text { volume }\left(\mathrm{cm}^{3} \mathrm{~g}^{-1}\right)\end{array}$ & $\begin{array}{l}\text { BJH average pore } \\
\text { diameter }(\mathrm{nm})\end{array}$ \\
\hline $\mathrm{SiO}_{2}$ & - & 272 & 0.91 & 10.2 \\
\hline $\mathrm{Ce} / \mathrm{SiO}_{2}$ & 4.1 & 297 & 0.82 & 5.2 \\
\hline $\mathrm{Sn} / \mathrm{SiO}_{2}$ & 4.6 & 366 & 0.56 & 6.7 \\
\hline
\end{tabular}

of active components from the solid. As the best results in the synthesis of trans-carveol and trans-sobrerol from $\alpha$-pinene oxide were previously obtained in dimethylacetamide (DMA) and acetone solutions, respectively [9], we initiated our study with the $\mathrm{Ce} / \mathrm{SiO}_{2}$ and $\mathrm{Sn} / \mathrm{SiO}_{2}$ catalysts using these compounds as solvents.

$\alpha$-Pinene oxide was relatively stable in DMA at $140{ }^{\circ} \mathrm{C}$. In a blank reaction, only a $5 \%$ conversion was observed for $20 \mathrm{~h}$ (Table 2 , run 1). To our surprise, pure silica, which was prepared by the same procedure as $\mathrm{Ce} / \mathrm{SiO}_{2}$ and $\mathrm{Sn} / \mathrm{SiO}_{2}$, promoted the isomerization of $\alpha$-pinene oxide at a reasonable rate with high selectivity for transcarveol. In the presence of only $2 \mathrm{wt} \%$ of $\mathrm{SiO}_{2}$ in DMA, a nearly complete conversion of $\alpha$-pinene oxide was attained for $10 \mathrm{~h}$ resulting in trans-carveol in $70 \%$ selectivity (Table 2, run 2 ). It is important that only two minor products have been observed in detectable amounts, i.e., campholenic aldehyde and trans-pinocarveol, which are also valuable compounds. A combined selectivity for campholenic aldehyde, trans-carveol and trans-pinocarveol was as high as $97 \%$.

The sol-gel silica-included cerium catalyst, $\mathrm{Ce} / \mathrm{SiO}_{2}$, promoted much faster reaction than pure silica giving a similar product distribution (Table 2, run 3 and run 2). In run 4, trans-carveol was formed in nearly $70 \%$ yield. However, in a further study it has been shown that the reaction is not truly heterogeneous and the catalyst loses the activity due to leaching of active components. The $\mathrm{Ce} / \mathrm{SiO}_{2}$ catalyst recovered from the reaction mixture after run 3 by filtration and reused in run 5 showed much lower activity (52\% vs. $98 \%$ conversion in $3 \mathrm{~h}$ ) than during the first use (Table 2). Although in the third reaction cycle (Table 2 , run 6 ) the activity of the catalyst did not decrease further, the rate of the reaction with the spent catalyst was nearly the same as that with pure silica. Whereas the fresh catalyst resulted in a virtually complete conversion for $3 \mathrm{~h}$ (Table 2 , run 3 ), in recycling experiments as well as with pure silica, only ca. $60 \%$ of the substrate was converted for the same reaction time (Table 2 , runs 2, 5 and 6). Moreover, when the catalyst was filtered off at the reaction temperature, the filtrate continued to convert $\alpha$-pinene oxide. Thus, the $\mathrm{Ce} / \mathrm{SiO}_{2}$ catalyst releases active components to the DMA solution and the process is not truly heterogeneous.

The sol-gel silica-included tin catalyst, $\mathrm{Sn} / \mathrm{SiO}_{2}$, also promoted the isomerization of $\alpha$-pinene oxide, with the reaction being even faster than that over $\mathrm{Ce} / \mathrm{SiO}_{2}$ (Table 2, cf. runs 3 and 7). The main reaction product was also trans-carveol obtained in a $70 \%$ yield. The combined yield for trans-carveol and campholenic aldehyde reached $90 \%$ in this reaction. However, leaching experiments with the tin catalyst also showed disappointing results. In run 8 (Table 2 ), which was performed under the same conditions as run 7 , the catalyst was removed by filtration after $0.5 \mathrm{~h}$ at the reaction temperature and the filtrate was allowed to react further. As it can be seen, the reaction keeps going in the absence of the solid, albeit at a lower rate. Thus, both the $\mathrm{Ce} / \mathrm{SiO}_{2}$ and $\mathrm{Sn} / \mathrm{SiO}_{2}$ materials are not stable in DMA solutions under the reaction conditions and release active components to the solution.

In a further study, we decided to use cerium and tin chlorides as soluble homogeneous catalysts for the isomerization of $\alpha$-pinene oxide in DMA. These compounds could represent cheaper alternatives for the homogeneous PW catalyst for the synthesis of trans-carveol from $\alpha$-pinene oxide developed in our previous work

Table 2

Isomerization of $\alpha$-pinene oxide (1) in dimethylacetamide (DMA) solutions at $140{ }^{\circ} \mathrm{C}$ a

\begin{tabular}{|c|c|c|c|c|c|c|c|c|c|c|}
\hline \multirow[t]{2}{*}{ Run } & \multirow[t]{2}{*}{ Catalyst } & \multirow[t]{2}{*}{ Ce or Sn $(\mu \mathrm{mol})$} & \multirow[t]{2}{*}{ Substrate (mmol) } & \multirow[t]{2}{*}{ Time (h) } & \multirow[t]{2}{*}{ Conversion (\%) } & \multicolumn{3}{|c|}{ Product selectivity (\%) } & \multirow[t]{2}{*}{$\mathrm{TON}^{\mathrm{b}}$} & \multirow[t]{2}{*}{$\operatorname{TOF}^{\mathrm{b}}\left(\mathrm{h}^{-1}\right)$} \\
\hline & & & & & & 2 & 3 & 4 & & \\
\hline 1 & - & - & 0.8 & 20 & 5 & & & & & \\
\hline \multirow[t]{2}{*}{2} & $\mathrm{SiO}_{2}(0.12 \mathrm{~g})$ & - & 0.8 & 3 & 58 & & & & & \\
\hline & & & & 10 & 95 & 16 & 70 & 11 & & \\
\hline 3 & $\mathrm{Ce} / \mathrm{SiO}_{2}$ & 40 & 0.8 & 3 & 98 & 19 & 72 & 5 & & \\
\hline 4 & $\mathrm{Ce} / \mathrm{SiO}_{2}$ & 40 & 2.4 & 8 & 98 & 18 & 73 & 5 & & \\
\hline $5^{c}$ & $\mathrm{Ce} / \mathrm{SiO}_{2}$ & 40 & 0.8 & 3 & 52 & 19 & 69 & 7 & & \\
\hline $6^{\mathrm{d}}$ & $\mathrm{Ce} / \mathrm{SiO}_{2}$ & 40 & 0.8 & 3 & 58 & 20 & 70 & 6 & & \\
\hline \multirow[t]{2}{*}{7} & $\mathrm{Sn} / \mathrm{SiO}_{2}$ & 40 & 0.8 & 0.5 & 48 & & & & & \\
\hline & & & & 1.5 & 100 & 20 & 70 & 5 & & \\
\hline \multirow[t]{2}{*}{$8^{e}$} & $\mathrm{Sn} / \mathrm{SiO}_{2}$ & 40 & 0.8 & 0.5 & 52 & & & & & \\
\hline & & & & 3 & 92 & 20 & 71 & 6 & & \\
\hline 9 & $\mathrm{CeCl}_{3}$ & 20 & 0.8 & 2 & 100 & 17 & 42 & 36 & 40 & 20 \\
\hline $10^{\mathrm{f}}$ & $\mathrm{CeCl}_{3}$ & 20 & 0.8 & 4 & 100 & 16 & 41 & 38 & 40 & 10 \\
\hline 11 & $\mathrm{SnCl}_{2}$ & 20 & 0.8 & 0.5 & 100 & 16 & 70 & 10 & 40 & 80 \\
\hline $12^{\mathrm{f}}$ & $\mathrm{SnCl}_{2}$ & 20 & 0.8 & 1 & 100 & 15 & 68 & 12 & 40 & 40 \\
\hline 13 & $\mathrm{SnCl}_{2}$ & 20 & 1.6 & 1 & 100 & 19 & 62 & 15 & 80 & 80 \\
\hline 14 & $\mathrm{SnCl}_{2}$ & 5 & 1.6 & 1.5 & 100 & 17 & 65 & 15 & 320 & 213 \\
\hline \multirow[t]{3}{*}{$15^{g}$} & $\mathrm{SnCl}_{2}$ & 5 & 1.6 & 1.5 & 98 & 17 & 62 & 17 & 634 & 210 \\
\hline & & & 1.6 & 2.5 & 95 & 19 & 64 & 15 & 938 & 122 \\
\hline & & & 1.6 & 4 & 90 & 18 & 60 & 19 & 1226 & 72 \\
\hline
\end{tabular}

a DMA $5 \mathrm{~mL}$, solid catalyst ca. 2.0-2.5 wt\%. Conversion and selectivity were determined by GC.

b TON - moles of the substrate converted/moles of Ce or Sn. TOF - the average turnover frequency.

c The catalyst was re-used after run 3 .

d The catalyst was re-used after run 5 .

e The catalyst was removed by filtration after $0.5 \mathrm{~h}$ at the reaction temperature and the filtrate was allowed to react further.

f $120^{\circ} \mathrm{C}$.

g After run 14, three fresh portions of the substrate (1.6 mmol each) were added consequently, each one after the nearly complete conversion of the previous portion. Total TON is given. 
Table 3

Isomerization of $\alpha$-pinene oxide ( $\mathbf{1})$ in acetone solutions. ${ }^{\mathrm{a}}$

\begin{tabular}{|c|c|c|c|c|c|c|c|c|c|}
\hline \multirow[t]{2}{*}{ Run } & \multirow[t]{2}{*}{ Catalyst } & \multirow[t]{2}{*}{ Ce or $\operatorname{Sn}(\mu \mathrm{mol})$} & \multirow[t]{2}{*}{ Substrate (mmol) } & \multirow[t]{2}{*}{$T\left({ }^{\circ} \mathrm{C}\right)$} & \multirow[t]{2}{*}{ Time (min) } & \multirow[t]{2}{*}{ Conversion (\%) } & \multicolumn{3}{|c|}{ Product selectivity (\%) } \\
\hline & & & & & & & 2 & 3 & 5 \\
\hline 1 & - & - & 0.8 & 25 & 300 & 5 & & & \\
\hline 2 & $\mathrm{SiO}_{2}(0.12 \mathrm{~g})$ & - & 0.8 & 25 & 180 & 84 & 19 & 10 & 69 \\
\hline 3 & $\mathrm{SiO}_{2}(0.12 \mathrm{~g})$ & - & 0.8 & 40 & 180 & 97 & 16 & 13 & 70 \\
\hline 4 & $\mathrm{Ce} / \mathrm{SiO}_{2}$ & 40 & 0.8 & 25 & 30 & 96 & 14 & 12 & 72 \\
\hline 5 & $\mathrm{Ce} / \mathrm{SiO}_{2}$ & 40 & 0.8 & 40 & 30 & 100 & 21 & 11 & 65 \\
\hline 6 & $\mathrm{Ce} / \mathrm{SiO}_{2}$ & 40 & 0.8 & 5 & 90 & 100 & 18 & 10 & 70 \\
\hline $7^{b}$ & $\mathrm{Ce} / \mathrm{SiO}_{2}$ & 20 & 0.8 & 25 & 180 & 98 & 16 & 10 & 72 \\
\hline 8 & $\mathrm{Sn} / \mathrm{SiO}_{2}$ & 20 & 0.8 & 25 & 15 & 100 & 17 & 11 & 70 \\
\hline 9 & $\mathrm{Sn} / \mathrm{SiO}_{2}$ & 10 & 0.8 & 25 & 15 & 100 & 16 & 10 & 72 \\
\hline 10 & $\mathrm{Sn} / \mathrm{SiO}_{2}$ & 10 & 1.6 & 25 & 60 & 95 & 17 & 11 & 72 \\
\hline \multirow[t]{2}{*}{$11^{\mathrm{b}}$} & $\mathrm{Sn} / \mathrm{SiO}_{2}$ & 10 & 0.8 & 25 & 30 & 43 & & & \\
\hline & & & & & 180 & 80 & 20 & 14 & 63 \\
\hline
\end{tabular}

a Conditions: acetone $5 \mathrm{~mL}$, solid catalyst $0.5-3.0 \mathrm{wt} \%$. Conversion and selectivity were determined by GC.

b Water was added (1 vol\%).

[9]. Representative results are shown in Table 2. In the presence of $\mathrm{CeCl}_{3}(0.025 \mathrm{eq})$, the reaction occurred smoothly resulting in a complete conversion for $2-4 \mathrm{~h}$ depending on the reaction temperature (Table 2, runs 9 and 10). However, the product distribution was different from that observed in the heterogeneous system, in which trans-pinocarveol was detected only as a minor product (ca. $5 \%$ ). In the cerium catalyzed homogeneous reaction, trans-carveol and trans-pinocarveol were formed in comparable amounts in ca. $40 \%$ selectivity each, with campholenic aldehyde being a main minor product (ca. 15\%). The system is synthetically useful considering a high combined yield for three expensive fragrance compounds (95\%), low catalyst to substrate ratio and possibility to re-use the catalyst solution in DMA after the extraction of the products with hydrocarbon solvent, e.g., hexane. In most of the reported systems, the main product of the $\alpha$-pinene oxide isomerization (usually campholenic aldehyde) was obtained along with numerous side products $[3,7]$.

The conversion of $\alpha$-pinene oxide in the solutions of $\mathrm{SnCl}_{2}$ $(0.025 \mathrm{eq})$ was very fast, much faster than with $\mathrm{CeCl}_{3}$. The tin catalyzed reactions showed four times higher average turnover frequencies (TOFs) than those catalyzed by cerium (Table 2 , runs 11 and 12 vs. runs 9 and 10) and gave ca. 95\% combined yield for campholenic aldehyde, trans-carveol and trans-pinocarveol, with trans-carveol accounting for ca. $70 \%$ of the mass balance. Aiming to improve the catalyst efficiency in terms of turnover number (TON) we increased the substrate concentration and then decreased the catalyst amounts (Table 2, runs 13 and 14). In run 14, TON of 320 per mol of Sn and 65\% selectivity to trans-carveol were obtained. After run 14, three fresh portions of the substrate ( $1.6 \mathrm{mmol}$ each) were added consequently, each one after the nearly complete conversion of the previous portion. The total TON of 1226 per mol of Sn was obtained in this reaction, which gave campholenic aldehyde, trans-carveol and trans-pinocarveol in a nearly quantitative combined yield (Table 2, run 15).

Thus, in the DMA solutions, tin and cerium catalysts as well as pure silica promote the isomerization of $\alpha$-pinene oxide giving mainly trans-carveol, with only two minor products: campholenic aldehyde and trans-pinocarveol.

In a further study, we have tested the prepared $\mathrm{Ce} / \mathrm{SiO}_{2}$ and $\mathrm{Sn} / \mathrm{SiO}_{2}$ materials for the isomerization of $\alpha$-pinene oxide in acetone solutions. Representative data are collected in Table 3. In this case, much more promising results in terms of catalyst leaching were obtained, which allowed the development of truly heterogeneous processes for the synthesis of highly valuable trans-sobrerol. In a blank reaction with no catalyst added, only $5 \%$ conversion was observed for $6 \mathrm{~h}$ at room temperature (Table 3, run 1 ). In the presence of pure silica, $\alpha$-pinene oxide undergoes a relatively fast isomerization even at room temperature (Table 3 , run 2 ). At $40^{\circ} \mathrm{C}$ with pure silica, a nearly complete conversion was achieved for $3 \mathrm{~h}$ giving trans-sobrerol in ca. 70\% yield (Table 3, run 3).

With the $\mathrm{Ce} / \mathrm{SiO}_{2}$ catalyst, the reaction showed a similar product distribution; however, it was much faster than that with pure silica (Table 3, runs 2 and 3 vs. runs 4 and 5). For the comparison purpose, the same mass amounts of $\mathrm{SiO}_{2}$ and $\mathrm{Ce} / \mathrm{SiO}_{2}$ were used in these runs. In an attempt to change the reaction selectivity, we decreased the reaction temperature; however, it did not affect significantly the reaction pathways (Table 3 , run 6 ). Then, we have added water ( $1 \mathrm{vol} \%$ ) to the system trying to increase the yield of the main product, trans-sobrerol, whose formation requires water (Table 3, run 7). However, we did not succeed: the best obtained yield of transsobrerol was nearly $70 \%$. It is important to note that only two minor products have been observed in detectable amounts along with trans-sobrerol, i.e., campholenic aldehyde and trans-carveol, with a total yield for all three valuable compounds being almost quantitative.

In the presence of the $\mathrm{Sn} / \mathrm{SiO}_{2}$ material, the reaction was very fast as the analysis of the first aliquot taken after 15 min showed a complete conversion (Table 3, runs 8 and 9). Further, the amount of the catalyst was decreased whereas the substrate concentration was increased (Table 3, run 10). In this run with only $0.5 \mathrm{wt} \%$ of the catalyst, trans-sobrerol was obtained in ca. $70 \%$ yield. Considering that for the formation of trans-sobrerol water is needed we have added $1 \mathrm{vol} \%$ of water to the system (Table 3 , run 11). However, the presence of extra amounts of water resulted in no changes in the product distribution, with the reaction rate being decreased significantly (Table 3 , run 11 vs. run 9). It should be mentioned that, generally, water was not added to the reaction system. The amount of hydration water present in the solid catalysts and commercial $\alpha$-pinene oxide and acetone was sufficient for the formation of sobrerol. In addition, water could be present inside $\mathrm{SiO}_{2}$ pores of the catalysts which had a pore volume of $0.56-0.91 \mathrm{~cm}^{3} \mathrm{~g}^{-1}$.

The leaching of active components from the $\mathrm{Ce} / \mathrm{SiO}$ and $\mathrm{Sn} / \mathrm{SiO}$ materials under the reaction conditions was verified in special experiments. After runs 5 and 9 (Table 3 ), the catalysts were filtered off at the reaction temperature to avoid re-adsorption of leached metal ions onto the solid support. Then, the filtrates were recharged with fresh substrate and allowed to react further. No conversion of $\alpha$-pinene oxide was observed after catalyst removing, providing strong evidence in support of heterogeneous catalysis. Thus, the reaction solutions contained no significant amounts of active species and the activity of both sol-gel silica-included catalysts, $\mathrm{Ce} / \mathrm{SiO}_{2}$ and $\mathrm{Sn} / \mathrm{SiO}_{2}$, was due to the active species immobilized in the mesoporous silica framework. The behavior of both spent catalysts recovered after runs 5 and 9 (Table 3 ) with fresh substrate was nearly the same as in the original reactions. 


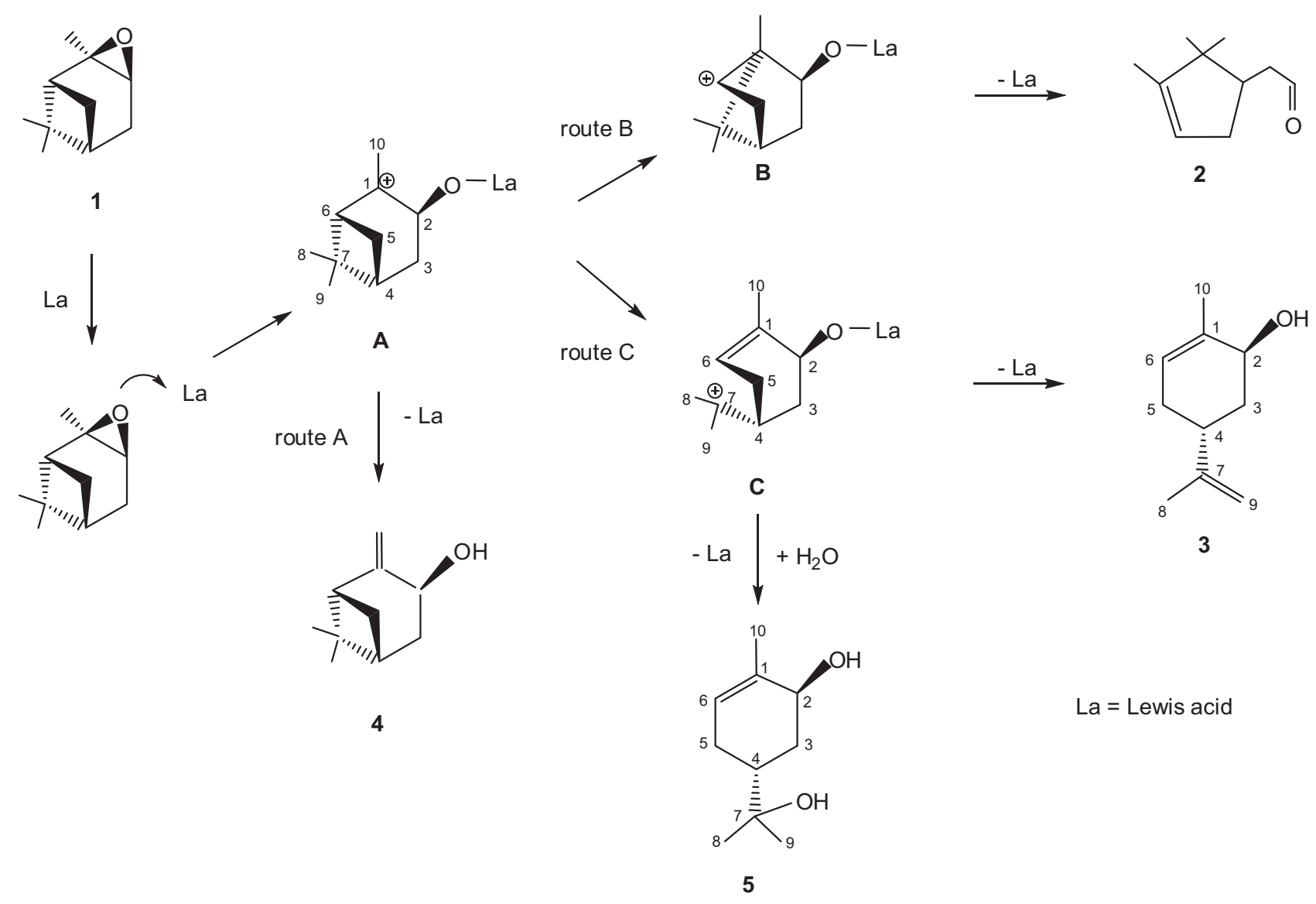

Scheme 2. Schematic representation of the Lewis acid-catalyzed transformations of $\alpha$-pinene-oxide.

A schematic representation of the Lewis acid-catalyzed transformations of $\mathbf{1}$ into products $\mathbf{2 - 5}$ is given in Scheme 2. In this scheme, "La" represents Lewis acid active sites on the catalyst surface (cationic cerium and tin species) or in the solution $\left(\mathrm{Ce}^{3+}\right.$ and $\mathrm{Sn}^{2+}$ ions). The activation of the epoxide moiety by the acidic sites induces epoxy ring opening and initially gives carbenium ion $\mathbf{A}$. The latter can undergo several competing processes. First, it can give directly, without any isomerization, trans-pinocarveol $\mathbf{4}$ through a hydrogen shift from the C-10 methyl group to the oxygen atom. The hydrogen abstraction and transfer could be assisted in our system by the basic solvent, DMA. In the $\mathrm{CeCl}_{3} / \mathrm{DMA}$ system, transpinocarveol accounts for ca. $40 \%$ of the mass balance. In addition, carbenium ion $\mathbf{A}$ can rearrange into carbenium ions $\mathbf{B}$ or $\mathbf{C}$ through the movement of the pair of electrons from the same carbon-carbon $\sigma$-bond to either C-7 or C-6, respectively. Carbocation B gives campholenic aldehyde 2 through the C-1-C-2 bond cleavage, whereas carbocation $\mathbf{C}$ forms trans-carveol $\mathbf{3}$ through the proton shift from the C-9 methyl group. The latter process could also be assisted by the basic solvent (in DMA) and/or by the oxygen atoms from the $\mathrm{SiO}_{2}$ support acting as Lewis basic sites (in both acetone and DMA solvents).

The results obtained in the present work showed that a solvent nature can profoundly affect the reaction pathways and product composition. This observation allowed us to develop efficient syntheses of various compounds from $\alpha$-pinene oxide through the choice of the appropriate solvent. As it can be seen in Scheme 2, relative amounts of trans-pinocarveol $\mathbf{4}$, the product with a bicyclic non-isomerized pinane skeleton, and monocyclic compounds 2, 3 and $\mathbf{5}$ depend on the balance between the hydrogen transfer in cation $\mathbf{A}$ (route $\mathrm{A}$ ) and its isomerization in cations $\mathbf{B}$ and $\mathbf{C}$ (routes $B$ and $C$ ). Trans-pinocarveol was formed in significant amounts in
DMA solutions but not in acetone. Both, DMA and acetone are highly polar solvents; however, DMA has much higher basicity $\left(\mathrm{pK}_{\mathrm{a}} \approx-0.5\right.$ for DMA $\mathrm{H}^{+}$vs. $\mathrm{pK}_{\mathrm{a}} \approx-7$ for $\left(\mathrm{CH}_{3}\right)_{2} \mathrm{COH}^{+}$[17]). Therefore, it seems reasonable that carbocation $\mathbf{A}$ is more prompted to undergo a proton shift (route A) before its isomerization (routes B and C) in DMA, which has relatively strong proton acceptor properties, than in less basic acetone.

On the other hand, the ratio between aldehyde $\mathbf{2}$ and paramenthenic compounds $\mathbf{3}$ and $\mathbf{5}$ is influenced by the balance between two different rearrangements of carbeniun ion $\mathbf{A}$ : into cyclopentanic cation B (route B) and into para-menthenic cation $\mathbf{C}$ (route $\mathbf{C}$ ). Tertiary carbeniun ion $\mathbf{C}$ is more thermodynamically stable than secondary carbenium ion $\mathbf{B}$; therefore, the formation of aldehyde $\mathbf{2}$ should be kinetically controlled. Carbenium ion $\mathbf{C}$ can give trans-carveol $\mathbf{3}$ or undergo a nucleophilic attack by water to form trans-sobrerol $\mathbf{5}$.

In the previous work [9], we have found that the increase in both solvent basicity and polarity strongly prejudices route $\mathrm{B}$ to aldehyde $\mathbf{2}$, whereas it favors route $C$ to para-menthenic products. For example, in cyclohexane, a non-polar non-basic solvent, the main reaction product was campholenic aldehyde 2 . A high ion-solvating ability of polar solvents could result in stabilization of cation $\mathbf{A}$ favoring its rearrangement into more stable tertiary carbenium ion $\mathbf{C}$. On the other hand, the distribution between paramenthenic products $\mathbf{3}$ and $\mathbf{5}$ depends on solvent basicity rather than on its polarity. In basic and polar DMA, where the proton transfer in $\mathbf{C}$ can be assisted by the solvent, the formation of trans-carveol $\mathbf{3}$ occurs faster than the formation of trans-sobrerol $\mathbf{5}$ and becomes the preferable reaction pathway.

It should be mentioned that the results obtained with different catalysts (PW, $\mathrm{Ce}, \mathrm{Sn}$ and $\mathrm{SiO}_{2}$ ) clearly show that the balance 
between the reaction pathways leading to different products from $\alpha$-pinene oxide is mostly determined by the nature of the solvent rather than by the nature of the catalyst or other reaction variables. However, further studies should be targeted toward clarifying the effect of solvent polarity and bacisity on the acidity of the catalyst surface, which should also be considered for understanding the reaction selectivity.

\section{Conclusions}

Chemoselectivity of the isomerization of $\alpha$-pinene oxide can be controlled through the choice of the solvent, whose polarity and basicity determine the reaction pathways. The heterogeneous process for the synthesis of highly valuable trans-sobrerol has been developed using weakly basic acetone as a solvent and silica-included tin and cerium materials as easily recoverable catalysts. Even pure silica alone can be used as a heterogeneous catalyst in this reaction, albeit less effective in terms of catalytic activity. On the other hand, in dimethylacetamide, which is much more basic, the reaction is essentially directed to transcarveol, regardless of the catalyst. The sol-gel Sn/SiO 2 and $\mathrm{Ce} / \mathrm{SiO}{ }_{2}$ materials showed high activity and selectivity for trans-carveol; however, the filtrate solutions promoted the isomerization of $\alpha$ pinene oxide after the catalyst removal. Due to these leaching problems, the synthesis of trans-carveol was performed using dissolved $\mathrm{CeCl}_{3}$ or $\mathrm{SnCl}_{2}$ as homogeneous catalysts or pure silica as a heterogeneous catalyst. The developed methods are among few examples of the selective synthesis of other than campholenic aldehyde compounds from $\alpha$-pinene oxide through its acid-catalyzed isomerization.

\section{Acknowledgments}

Financial support from the CNPq, CAPES, FAPEMIG and INCTCatálise (Brazil) is gratefully acknowledged.

\section{References}

[1] H. Mimoun, Chimia 50 (1996) 620-625.

[2] C. Chapuis, D. Jacoby, Appl. Catal. A 221 (2001) 93-117.

[3] W.F. Hölderich, J. Röseler, G. Heitmann, A.T. Liebens, Catal. Today 37 (1997) 353-366.

[4] G. Orloff, B. Winter, C. Fehr, in: P.M. Muller, D. Lamparsky (Eds.), Perfumes, Art, Science \& Technology, Elsevier, New York, 1991, p. 287.

[5] P.J. Kunkeler, J.C. van der Waal, J. Bremmer, B.J. Zuurdeeg, R.S. Downing, H. van Bekkum, Catal. Lett. 53 (1998) 135-138.

[6] L. Alaerts, E. Séguin, H. Poelman, F. Thibault-Starzyk, P.A. Jacobs, D.E. De Vos, Chem. Eur. J. 12 (2006) 7353-7363.

[7] N. Ravasio, F. Zaccheria, A. Gervasini, C. Messi, Catal. Commun. 9 (2008) $1125-1127$.

[8] K.A. da Silva Rocha, I.V. Kozhevnikov, E.V. Gusevskaya, Appl. Catal. A 294 (2005) $106-110$.

[9] K.A. da Silva Rocha, J.L. Hoehne, E.V. Gusevskaya, Chem. Eur. J. 14 (2008) 6166-6172.

[10] W.B. Motherwell, M.J. Bingham, J. Pothier, Y. Six, Tetrahedron 60 (2004) 3231-3241.

[11] Q. Wang, Y. Li, Q. Chen, Synth. Commun. 33 (2003) 2125-2134.

[12] J. Kaminska, M.A. Schwegler, A. Hoefnagel, H. van Bekkum, Recl. Trav. Chim. Pays-Bas 111 (1992) 432-437.

[13] L.A. Parreira, L. Menini, J.C. da Cruz Santos, E.V. Gusevskaya, Adv. Synth. Catal 352 (2010) 1533-1538.

[14] K.A. da Silva Rocha, N.V.S. Rodrigues, I.V. Kozhevnikov, E.V. Gusevskaya, Appl. Catal. A 374 (2010) 87-94.

[15] M.G. Speziali, V.V. Costa, P.A. Robles-Dutenhefner, E.V. Gusevskaya, Organometallics 28 (2009) 3186-3192.

[16] P.A. Robles-Dutenhefner, K.A. da Silva Rocha, E.M.B. Sousa, E.V. Gusevskaya, J. Catal. 265 (2009) 72-79.

[17] J. March, Advanced Organic Chemistry: Reactions, Mechanisms and Structures, fourth ed., Wiley, New York, 1992, p. 250. 